between an acid in aqueous solution and an alloy of boron gives very poor yields and is long and tedious. Schlesinger and Burg have described ( $J$. Amer. Chem. Soc., Dec. 1931) a method for the preparation of diborane, $\mathrm{B}_{2} \mathrm{H}_{6}$, which consists in the reduction of gaseous boron trichloride by hydrogen in an electric arc discharge at low pressure. Boron, various nonvolatile hydrides and large yields of an unstable spontaneously inflammable substance, considered from its reactions to be $\mathrm{B}_{2} \mathrm{H}_{5} \mathrm{Cl}$, are also formed. The last-named compound, however, on warming decomposes and gives a further quantity of diborane : $6 \mathrm{~B}_{2} \mathrm{H}_{5} \mathrm{Cl}=5 \mathrm{~B}_{2} \mathrm{H}_{6}+2 \mathrm{BCl}_{3}$. When diborane containing a small quantity of hydrogen chloride is heated at $120^{\circ}-130^{\circ}$ a considerable proportion is converted into the more stable pentaboron hydride $\mathrm{B}_{5} \mathrm{H}_{9}$.

Aromatic Nitroso-Compounds in Solution.-The apparent molecular weights of nitrosobenzene and a number of its derivatives were determined in dilute solutions, mostly in benzene, by Bamberger and coworkers, and the results seemed to indicate that the state was unimolecular. Hammick has shown that these results are ambiguous, and has described now experiments with co-workers on nitrosobenzene and several substituted products (J.Chem. Soc., Dec. 1931). The results show that nitrosobenzene itself and its meta- and para-derivatives are associated in dilute solution. In the case of mono- and di-ortho-substituents a steric effect was detected. The degree of association of nitrosobenzene itself is quite small, about $2 \cdot 4$ per cent, but in some of the derivatives large associations were found, such as 38.2 per cent for o-nitronitrosobenzene and $77 \cdot 3$ per cent for nitrosomesitylene. In the di-ortho-substituted derivatives there is enormously enhanced stability of the bimolecular form. The first step in association in the case of nitrosobenzene is thought to be dipole association, although it is considered unlikely that the bimolecular forms of nitroso-compounds are simply dipole aggregates, and the dipole aggregate is thought to pass into an oxyazoxy-structure.

A Compound of Nitrobenzene and Sulphuric Acid.A compound, $\mathrm{C}_{6} \mathrm{H}_{5} \mathrm{NO}_{2}, \mathrm{H}_{2} \mathrm{SO}_{4}$, separates on cooling an equimolecular mixture of the two components, the melting point, $11 \cdot 6^{\circ}$, being higher than that of either component. Masson has published ( ${ }^{\mathrm{I}} \mathrm{I}$. Chem. Soc., Dec. 1931) a complete freezing-point diagram for the two substances, which shows a well-defined maximum corresponding with the equimolecular compound and two eutectics, so that probably this is the only compound formed. The compound forms colourless needles at low temperatures, but at room temperature is an oily liquid which is surprisingly sluggish towards water and does not liberate much heat when decom. posed by it. In sulphuric acid it forms solutions which conduct better than the acid alone, so that the compound is regarded as an electroly te $\left(\mathrm{C}_{6} \mathrm{H}_{5} \cdot \mathrm{NO}_{2} \cdot \mathrm{H}\right)^{+}$ $\left(\mathrm{HSO}_{4}\right)^{-}$, the hydrogen ion or proton being co-ordinated with one of the oxygen atoms of the nitro-group. The attachment of the positive hydrogen ion to the nitrogroup should promote meta-substitution in the same way as happens with an arylammonium ion. The stability of the compound suggests, indeed, that in the dinitration of nitrobenzene it may well be the positive nitrobenzene-hydrogen ion, rather than the neutral nitrobenzene molecule, which is the main organic reagent and thus the source of the preponderating metaderivative.

\title{
Astronomical Topics
}

Reinmuth's New Object.-Dr. Kahrstedt has published elements of this object in Rech. Inst. Circ. 534, as follows :

$\left.\begin{array}{rlll}\mathrm{T} & 1933 & \text { Oct. } 28 \cdot 2854 & \text { U.T. } \\ \omega & 96^{\circ} & 34^{\prime} & 0^{\prime \prime} \\ \Omega & 45 & 46 & 21 \\ i & 31 & 14 & 21\end{array}\right\} 1932 \cdot 0$

The orbit is still very uncertain, the arc being short. The object appears to be of the same type as comet Schwassmann-Wachmann (1), but with a somewhat smaller orbit that comes inside that of Jupiter at perihelion. The orbit makes a near approach to that of Jupiter at the descending node. The above cicular gives an ephemeris for February and March, but as the comet is fainter than mag. 15, it is not given here.

Rech. Inst. Circ. No. 540, dated Jan. 30, states that Prof. M. Wolf has changed his opinion about the object, and no longer considers that it is nebulous : it may, therefore, be a minor planet, not a comet; but even in this case it deserves attention, as its orbit appears to be of an unusual type.

The Light Variation of Cepheids.-Meddelande of Lund Observatory, Series I., No. 128, contains a study by Dr. K. Lundmark of the correlations between period and magnitude and between period and amplitude of light-change in the Cepheid variables. He includes in his study the stars in the Magellanic Clouds, the nearest two spiral nebulæ, the globular clusters, also galactic Cepheids. Graphs are given for the Cepheids in all these different regions; they agree in showing a close correlation between mag. nitude and logarithm of period, when the maximum magnitude is used; there is greater departure from a linear graph when the minimum magnitudes are used. There are also clearly recognisable correlations between amplitude and magnitude, but the graphs are more curved. It is noted that Dr. Hertzsprung found little trace of this last correlation in the stars near Eta Carinæ; but probably the stars that he was discussing were at very different distances.

Dr. Lundmark uses his adopted curves to obtain new estimates of the distances in light-years of the various objects; of course, they do not differ much from those already published. $\mathrm{He}$ finds for the Greater Cloud 95,000 light-years, for the Lesser one 112,000 light-years, for N.G.C. 6822690,000 lightyears, for the Andromeda nebula 840,000 light-years, for Messier 33860,000 light-years; the distance between the last two objects would be 224,000 light. years.

Rocznik Astronomiczny, Cracow Observatory.No, 10 of this annual publication, edited by T. Banachiewicz, contains the ephemerides of variable stars which the above observatory undertook to compute, with the aid of grants from the I.A.U. and the Polish Minister of Education.

It includes 44 eclipsing variables with period less than $18^{\mathrm{h}}, 219$ with period between $18^{\mathrm{h}}$ and $10^{\mathrm{d}}$, and 26 with periods exceeding 10d. Elements, but not ephemerides, are given for 32 other stars. The ephemerides are heliocentric. For reasons of economy, stars south of Decl. $-23^{\circ}$ are omitted this year, also the index of ephemeris-stars.

The volume also contains certain coefficients for calculating precessions and the times of occultations of stars by the moon for five observatories in Poland. The explanations, and the headings of tables, are in flexible Latin, which is easy to read.

No. 3250, VoL. 129] 